\title{
EXOTIC METRICS ON IMMERSED SURFACES
}

\author{
JOSEPH A. WOLF ${ }^{1}$
}

To A. A. Albert on his sixtieth birthday

1. Introduction. In a series of articles ([1], [2], [3], [4]), Tilla Klotz studied immersed surfaces by examining riemannian metrics constructed as linear combinations $s_{1} I+s_{2} I I+s_{3} I I I$ of the fundamental forms of the immersion. Here we study the Gauss curvature of pseudo-riemannian metrics $s_{1} I+s_{2} I I+s_{3} I I I$. If $s_{i}$ are constants, and if mean and Gauss curvature satisfy $s_{1}+s_{2} H+s_{3} K=0$, then we show that the metric $s_{1} I+s_{2} I I+s_{3} I I I$ is flat where it is nondegenerate. In particular we prove that $I I$ is a flat Lorentz metric on the complement of the umbilic set of a minimal surface.

2. The structure equations. Let $S$ be a pseudo-riemannian 2manifold with metric $d \nu^{2}$. This means that $S$ is a 2-dimensional differentiable manifold and $d \nu^{2}$ is a smooth ${ }^{2}$ family of nondegenerate inner products on the tangent planes of $S$. If the inner products are all positive definite, then $S$ is a riemannian 2-manifold. Given $x \in S$ we write $S_{x}$ for the tangent plane at $x$. If $X \in S_{x}$, then $d \nu_{x}^{2}$ denotes the inner product on $S_{x}$, and $\|X\|^{2}$ denotes $d \nu_{x}^{2}(X, X)$. Let $\left\{X_{1}, X_{2}\right\}$ be a moving frame on an open set $U \subset S$. This means that the $X_{i}$ are smooth tangent vector fields on $U$ which are linearly independent at every point. Then the "dual co-frame" is the pair $\left\{\theta^{1}, \theta^{2}\right\}$ of linear differential forms on $U$ defined by $\theta^{i}\left(a^{1} X_{1}+a^{2} X_{2}\right)=a^{i}$; the metric has local expression $d \nu^{2}=\sum_{i, j} g_{i j} \theta^{i} \otimes \theta^{j}$ where the "coefficients" are the functions $g_{i j}(x)=d \nu_{x}^{2}\left(X_{i x}, X_{j x}\right)$.

The moving frame $\left\{X_{1}, X_{2}\right\}$ is called orthonormal if $g_{i j}= \pm \delta_{i j}$. This means that $\left\|X_{i}\right\|^{2}=e_{i}= \pm 1$ and $d \nu^{2}\left(X_{1}, X_{2}\right) \equiv 0$, and it says that $d \nu^{2}=\sum_{i} e_{i} \theta^{i} \otimes \theta^{i}$. An obvious modification of the Gram-Schmidt process constructs an orthonormal moving frame from an arbitrary moving frame.

Let $\left\{X_{1}, X_{2}\right\}$ be an orthonormal moving frame on an open set $U \subset S$. Then the dual coframe $\left\{\theta^{1}, \theta^{2}\right\}$ is also called "orthonormal," and we have the signs $e_{i}=\left\|X_{i}\right\|^{2}= \pm 1$. New forms are defined on $U$ by

$$
\theta_{i}=e_{i} \theta^{i}
$$

Received by the editors December 14, 1965.

1 Research supported in part by National Science Foundation Grant GP 2439, in part by an Alfred P. Sloan Research Fellowship.

2 "Smooth" means "sufficiently differentiable." The reader can easily keep count. 
and we define functions $a_{i}(x)$ on $U$ by

$$
d \theta_{i}=a_{i} \theta_{1} \wedge \theta_{2} .
$$

Now the connection forms are the linear differential forms $\omega_{i j}$ defined on $U$ by

$$
-\omega_{12}=+\omega_{21}=e_{2} a_{1} \theta_{1}+e_{1} a_{2} \theta_{2}, \quad \omega_{11}=0=\omega_{22} .
$$

They are characterized by the structure equations

$$
d \theta^{i}=\sum_{j} \theta^{j} \wedge \stackrel{i}{\omega_{j}, \omega_{j}^{i}}=e_{i} \omega_{i j}, \quad \omega_{i j}+\omega_{j i}=0 .
$$

The connection forms $\omega_{i j}$ are specified by $\omega_{12}$, and the structure equations can be written

$$
d \theta_{1}=e_{2} \theta_{2} \wedge \omega_{12} \text { and } d \theta_{2}=\omega_{12} \wedge e_{1} \theta_{1} .
$$

Gauss curvature is a function $K(x)$ on $S$. In the notation above, it is defined on the open set $U \subset S$ by the equation

$$
d \omega_{12}=K \theta_{1} \wedge \theta_{2} .
$$

One can check [5, Theorem 2.2.1] that this defines $K$ independently of the choice of orthonormal moving frame, and we will note in Lemma 4.5 that it is equivalent to the classical definition for surfaces immersed in $R^{3}$.

3. Metrics associated to quadratic differential forms. $S$ denotes a fixed riemannian 2-manifold with (positive definite) metric $d \nu^{2}$, and we study the geometry of a smooth family $\Phi=\left\{\Phi_{x}\right\}_{x \in S}$ of inner products on the tangent planes of $S$. Eventually $S$ will be an immersed surface and $\Phi$ will be a linear combination of its fundamental forms.

If $x \in S$, then we diagonalize $\Phi_{x}$ relative to $d \nu_{x}^{2}$; so $\Phi_{x}$ has matrix

$$
\left(\begin{array}{cc}
f_{1}(x) & 0 \\
0 & f_{2}(x)
\end{array}\right)
$$

in some orthonormal basis of $S_{x}$. Generalizing the case where $\Phi$ is the second fundamental form of an immersion, we define

$f_{i}(x)$ : the principle $\Phi$-curvatures at $x$,

$H_{\Phi}(x)$ : the mean $\Phi$-curvature $\frac{1}{2}\left\{f_{1}(x)+f_{2}(x)\right\}$,

$K_{\Phi}(x)$ : the Gauss $\Phi$-curvature $f_{1}(x) \cdot f_{2}(x)$,

$\Phi$-elliptic point: point $x$ with $K_{\Phi}(x)>0$,

$\Phi$-parabolic point: point $x$ with $K_{\Phi}(x)=0$,

$\Phi$-hyperbolic point: point $x$ with $K_{\Phi}(x)<0$,

$\Phi$-umbilic: point $x$ with $f_{1}(x)=f_{2}(x)$. 
The set of all $\Phi$-umbilics, and the set of all $\Phi$-parabolic points, are closed in $S$. Thus the set

$$
S_{\Phi}=\{x \in S: x \text { is neither } \Phi \text {-umbilic nor } \Phi \text {-parabolic }\}
$$

is open in $S$, and $\Phi$ restricts to a pseudo-riemannian metric $d s_{\Phi}^{2}$ on $S_{\Phi}$. We will study the pseudo-riemannian manifold $S_{\Phi}$ with metric $d s_{\Phi}^{2}$.

3.2. Theorem. Let $x \in S_{\Phi}$. Then $x$ has an open neighborhood $U \subset S_{\Phi}$ which carries linear differential forms $\theta^{i}$ such that

(3.3) $d \nu^{2}=\theta^{1} \otimes \theta^{1}+\theta^{2} \otimes \theta^{2} \quad$ and $\quad d s_{\Phi^{2}}=f_{1} \theta^{1} \otimes \theta^{1}+f_{2} \theta^{2} \otimes \theta^{2}$ in $U$.

Define functions $r_{i}>0$ in $U$ by $r_{i}^{2}=\epsilon_{i} f_{i}, \epsilon_{i}= \pm 1$, so the $\phi^{i}=r_{i} \theta^{i}$ are $d s_{\Phi^{2}}^{2}$-orthonormal. Let $\omega_{12}$ and $\beta_{12}$ be the respective connection forms for $d \nu^{2}$ relative to $\left\{\theta^{1}, \theta^{2}\right\}$ and $d s_{\Phi}^{2}$ relative to $\left\{\phi^{1}, \phi^{2}\right\}$. Then

$$
\beta_{12}=-\left|f_{1} f_{2}\right|^{-1 / 2}\left\{\left(f_{1} a_{1}-\frac{1}{2} f_{1 ; 2}\right) \theta^{1}+\left(f_{2} a_{2}+\frac{1}{2} f_{2 ; 1}\right) \theta^{2}\right\}
$$

where $d f_{i}=\sum_{j} f_{i ; j} \theta^{j}$ and $\omega_{12}=-\left(a_{1} \theta^{1}+a_{2} \theta^{2}\right)$.

Proof. For the first assertion we choose an orthonormal frame $\left\{X_{1}, X_{2}\right\}$ on a neighborhood $U$ of $x$ such that $\Phi_{z}$ is diagonal relative to $\left\{X_{1 z}, X_{2 z}\right\}$ for every $z \in U$. Then (3.3) follows with $\left\{\theta^{1}, \theta^{2}\right\}$ dual to $\left\{X_{1}, X_{2}\right\}$ because $f_{1} \neq f_{2}$ throughout $S_{\Phi}$.

Define $a_{i}$ and $b_{i}$ by $d \phi_{i}=b_{i} \phi_{1} \wedge \phi_{2}$ and $d \theta_{i}=a_{i} \theta_{1} \wedge \theta_{2}$. Then (2.3) says

$$
\omega_{12}=-\left(a_{1} \theta_{1}+a_{2} \theta_{2}\right) \text { and } \beta_{12}=-\left(\epsilon_{2} b_{1} \phi_{1}+\epsilon_{1} b_{2} \phi_{2}\right) \text {. }
$$

Now compute

$$
\begin{aligned}
d \phi_{i} & =d\left(\epsilon_{i} \phi^{i}\right)=\epsilon_{i} d \phi^{i}=\epsilon_{i}\left(d r_{i} \wedge \theta^{i}+r_{i} d \theta^{i}\right), \\
\epsilon_{i} d r_{i} \wedge \theta^{i} & =\epsilon_{i} r_{i} d\left(\log r_{i}\right) \wedge \theta^{i}=d\left(\log r_{i}\right) \wedge \phi_{i} \\
& =\frac{1}{2} d\left(\log r_{i}^{2}\right) \wedge \phi_{i}=\frac{1}{2} r_{i}^{-2} d\left(\epsilon_{i} f_{i}\right) \wedge \phi_{i} \\
& =\frac{1}{2} f_{i}^{-1}\left(f_{i ; 1} \theta^{1}+f_{i ; 2} \theta^{2}\right) \wedge \phi_{i}=\frac{1}{2 f_{i}}\left\{\frac{\epsilon_{1}}{r_{1}} f_{i ; 1} \phi_{1}+\frac{\epsilon_{2}}{r_{2}} f_{i ; 2} \phi_{2}\right\} \wedge \phi_{i}, \\
\epsilon_{i} r_{i} d \theta^{i} & =\epsilon_{i} r_{i} d \theta_{i}=\epsilon_{i} r_{i} a_{i} \theta_{1} \wedge \theta_{2}=\epsilon_{i} \epsilon_{1} \epsilon_{2} \frac{r_{i} a_{i}}{r_{1} r_{2}} \phi_{1} \wedge \phi_{2} .
\end{aligned}
$$

Thus

$$
b_{1}=\frac{\epsilon_{2}}{r_{2}}\left\{a_{1}-\frac{f_{1 ; 2}}{2 f_{1}}\right\} \quad \text { and } \quad b_{2}=\frac{\epsilon_{1}}{r_{1}}\left\{a_{2}+\frac{f_{2 ; 1}}{2 f_{2}}\right\}
$$




$$
\begin{aligned}
\beta_{12} & =-\left\{\frac{1}{r_{2}}\left(a_{1}-\frac{f_{1 ; 2}}{2 f_{1}}\right) \phi_{1}+\frac{1}{r_{1}}\left(a_{2}+\frac{f_{2 ; 1}}{2 f_{2}}\right) \phi_{2}\right\} \\
& =-\left\{\frac{\epsilon_{1} r_{1}}{r_{2}}\left(\frac{2 f_{1} a_{1}-f_{1 ; 2}}{2 f_{1}}\right) \theta^{1}+\frac{\epsilon_{2} r_{2}}{r_{1}}\left(\frac{2 f_{2} a_{2}+f_{2 ; 1}}{2 f_{2}}\right) \theta^{2}\right\} \\
& =-\frac{1}{r_{1} r_{2}}\left\{\left(f_{1} a_{1}-\frac{1}{2} f_{1 ; 2}\right) \theta^{1}+\left(f_{2} a_{2}+\frac{1}{2} f_{2 ; 1}\right) \theta^{2}\right\} .
\end{aligned}
$$

The assertion follows from $f_{i}=\epsilon_{i} r_{i}^{2}$.

q.e.d.

A pseudo-riemannian 2-manifold is called flat if its Gauss curvature is identically zero.

3.5. Corollary. If $2 a_{1} f_{1}=f_{1 ; 2}$ and $2 a_{2} f_{2}+f_{2 ; 1}=0$, then $S_{\Phi}$ is flat.

4. Metrics defined by immersions. An immersed surface is a pair $(S, \nu)$ where $S$ is a two dimensional differentiable manifold and $\nu: S \rightarrow R^{3}$ is a differentiable map with nowhere vanishing Jacobian determinant. Thus $\nu(S)$ is a smooth surface in $R^{3}$ which has no singularities but may have self intersections. The inner products on the tangent planes of $\nu(S)$ define a riemannian metric $d \nu^{2} \equiv d \nu \cdot d \nu$ on $S$, and we view $S$ as a riemannian 2 -manifold with that metric.

Let $\xi$ be a smooth choice of unit normal to $\nu(S)$, defined over an open set $U \subset S$. Then we recall the classical quadratic differential forms

$$
\begin{aligned}
I & =d \nu \cdot d \nu, \text { first fundamental form } \\
I I & =d \nu \cdot d \xi, \text { second fundamental form } \\
I I I & =d \xi \cdot d \xi, \text { third fundamental form }
\end{aligned}
$$

Of course $I I$ is only defined up to sign unless we have an orientation on $S$. Principle, mean and Gauss curvature of $(S, \nu)$, and elliptic, parabolic, hyperbolic and umbilic points, are classically defined as in $\$ 3$ for the case $\Phi=I I$.

Let $\left\{v_{1}, v_{2}, v_{3}\right\}$ be a Darboux frame on an open set $U \subset S$. This means that $\left\{v_{1}, v_{2}\right\}$ is a moving orthonormal frame and $v_{3}$ is a smooth unit normal. Viewing $\nu$ as position vector, now

$$
d \nu=\theta^{1} v_{1}+\theta^{2} v_{2} \text { where }\left\{\theta^{1}, \theta^{2}\right\} \text { is dual to }\left\{v_{1}, v_{2}\right\} .
$$

We define forms $\omega_{j}^{i}$ on $S$ by

$$
d v_{j}=\sum_{i=1}^{3} \stackrel{i}{\omega_{j} v_{i}}
$$

Writing out $0=d(d \nu)$ and $0=d\left(d v_{j}\right)$, one has 


$$
d \theta^{i}=\sum_{j=1}^{2} \theta^{j} \wedge \omega_{j}^{i}, \quad 0=\sum_{j=1}^{2} \theta^{j} \wedge \omega_{j}^{3}, \quad d \omega_{j}^{i}=\sum_{k=1}^{3} \omega_{j}^{k} \wedge \omega_{k}^{i}
$$

As $\left\|v_{i}\right\|^{2}=1$ now $\omega_{i j}=\omega_{j}^{i}$, and differentiation of $v_{i} \cdot v_{j}=\delta_{i j}$ gives $\omega_{i j}+\omega_{j i}$ $=0$. Now (4.3) yields

$$
d \theta_{1}=\theta_{2} \wedge \omega_{12}, \quad d \theta_{2}=\omega_{12} \wedge \theta_{1}, \quad d \omega_{12}=\omega_{32} \wedge \omega_{13} ;
$$

and (2.4) shows that $\omega_{12}$ is the connection form.

4.5. Lemma. Let $k_{i}$ be the principle curvatures on $(S, \nu)$ and suppose that $x \in S$ is not an umbilic. Then $x$ has an open neighborhood $U \subset S$ which carries a Darboux frame $\left\{v_{1}, v_{2}, v_{3}\right\}$ in which

$$
I=\sum_{i=1}^{2} \theta^{i} \otimes \theta^{i}, \quad I I=\sum_{i=1}^{2} k_{i} \theta^{i} \otimes \theta^{i} \quad \text { and } \quad I I I=\sum_{i=1}^{2} k_{i}^{2} \theta^{i} \otimes \theta^{i} .
$$

In this frame $\omega_{3 i}=k_{i} \theta_{i}$, so Gauss curvature $K=k_{1} k_{2}$.

The result is standard. $x$ has a neighborhood $U_{1}$ of nonumbilics, which contains a smaller neighborhood $U$ carrying a smooth unit normal $v_{3}$. Order the $k_{i}$ on $U$ with $k_{1}>k_{2}$, and let $\left\{v_{1}, v_{2}\right\}$ give the corresponding principle directions. That constructs the Darboux frame, and $I$ and $I I$ have the required form. It follows that $d v_{3}=\sum_{i=1}^{2} k_{i} \theta^{i} v_{i}$, so $\omega_{3 i}=k_{\imath} \theta_{i}$ and $I I I$ has the required form. Now the structure equations give

$$
K \theta_{1} \wedge \theta_{2}=d \omega_{12}=\omega_{32} \wedge \omega_{13}=\omega_{31} \wedge \omega_{32}=k_{1} k_{2} \theta_{1} \wedge \theta_{2}
$$

so $K=k_{1} k_{2}$.

q.e.d.

4.6. TheOREM. Let $(S, \nu)$ be an immersed surface with principle curvatures $k_{i}$, mean curvature $H=\frac{1}{2}\left(k_{1}+k_{2}\right)$ and Gauss curvature $K=k_{1} k_{2}$. Let $s_{i}$ be differentiable functions on $S$ and define $\Phi$ to be the quadratic differential form $s_{1} I+s_{2} I I+s_{3} I I I$. Choose a Darboux frame $\left\{v_{1}, v_{2}, v_{3}\right\}$ satisfying Lemma 4.5 and define functions $a_{i}, k_{i ; j}$ and $s_{i ; j}$ by $d \theta^{i}$ $=a_{i} \theta^{1} \wedge \theta^{2}, d k_{i}=k_{i ; 1} \theta^{1}+k_{i ; 2} \theta^{2}$ and $d s_{i}=s_{i ; 1} \theta^{1}+s_{i ; 2} \theta^{2}$. If

$$
2 a_{1}\left(s_{1}+s_{2} H+s_{3} K\right)-\left(s_{1 ; 2}+s_{2 ; 2} k_{1}+s_{3 ; 2} k_{1}^{2}\right)=0
$$

and

$$
2 a_{2}\left(s_{1}+s_{2} H+s_{3} K\right)+\left(s_{1 ; 1}+s_{2 ; 1} k_{2}+s_{3 ; 1} k_{2}^{2}\right)=0,
$$

then $S_{\Phi}$ is flat.

Proof. Following Corollary 3.5, we find the condition for $2 a_{1} f_{1}$ 
$-f_{1 ; 2}=0=2 a_{2} f_{2}+f_{2 ; 1}$. Here Lemma 4.5 shows that $f_{i}=s_{1}+s_{2} k_{i}+s_{3} k_{i}^{2}$; thus

$$
f_{i ; j}=s_{1 ; j}+s_{2 ; j} k_{i}+s_{3 ; j} k_{i}^{2}+s_{2} k_{i ; j}+2 s_{3} k_{i} k_{i ; j}
$$

To evaluate this we compute

$$
\begin{aligned}
\left(a_{1} k_{1}-k_{1 ; 2}\right) \theta_{1} \wedge \theta_{2} & =d k_{1} \wedge \theta_{1}+k_{1} d \theta_{1}=d\left(k_{1} \theta_{1}\right)=d \omega_{13}=\omega_{23} \wedge \omega_{12} \\
& =k_{2} \theta_{2} \wedge \omega_{12}=a_{1} k_{2} \theta_{1} \wedge \theta_{2}
\end{aligned}
$$

and similarly

$$
\left(a_{2} k_{2}+k_{2 ; 1}\right) \theta_{1} \wedge \theta_{2}=d\left(k_{2} \theta_{2}\right)=a_{2} k_{1} \theta_{1} \wedge \theta_{2},
$$

so

$$
k_{1 ; 2}=a_{1}\left(k_{1}-k_{2}\right) \quad \text { and } \quad k_{2 ; 1}=a_{2}\left(k_{1}-k_{2}\right) .
$$

Combining (4.7) and (4.8) we have

$$
\begin{aligned}
& f_{1 ; 2}=s_{1 ; 2}+s_{2 ; 2} k_{1}+s_{3 ; 2} k_{1}^{2}+\left(s_{2}+2 s_{3} k_{1}\right) a_{1}\left(k_{1}-k_{2}\right), \\
& f_{2 ; 1}=s_{1 ; 1}+s_{2 ; 1} k_{2}+s_{3 ; 1} k_{2}^{2}+\left(s_{2}+2 s_{3} k_{2}\right) a_{2}\left(k_{1}-k_{2}\right) .
\end{aligned}
$$

It follows that

$$
\begin{aligned}
& 2 a_{1} f_{1}-f_{1 ; 2}=2 a_{1}\left(s_{1}+s_{2} H+s_{3} K\right)-\left(s_{1 ; 2}+s_{2 ; 2} k_{1}+s_{3 ; 2} k_{1}^{2}\right), \\
& 2 a_{2} f_{2}+f_{2 ; 1}=2 a_{2}\left(s_{1}+s_{2} H+s_{3} K\right)+\left(s_{1 ; 1}+s_{2 ; 1} k_{2}+s_{3 ; 1} k_{2}^{2}\right) .
\end{aligned}
$$

Now our assertion follows from Corollary 3.5.

q.e.d.

The most tractable special case is when the $s_{i}$ are constants. Then $s_{i ; j}=0$ and Theorem 4.6 simplifies to:

4.9. Theorem. Let $(S, \nu)$ be an immersed surface with mean curvature $H$ and Gauss curvature $K$. Let $s_{i}$ be constants and suppose

$$
s_{1}+s_{2} H+s_{3} K \equiv 0 \text { on } S \text {. }
$$

Then $S_{s_{1} I+s_{2} I I+s_{3} I I I}$ is flat.

Condition (4.10) specifies an interesting class of Weingarten surfaces, including the surfaces of constant mean curvature and the surfaces of constant Gauss curvature. For those surfaces we have:

4.11. CoRollary. Let $(S, \nu)$ be an immersed surface of mean curvature $H$ and Gauss curvature $K$, and let $b$ be a nonzero real number. 
1. $S_{-b H I+b I I}$ is the set of nonumbilic points of $S$. If $H$ is constant then $S_{-b H} I_{+b I I}$ is a flat Lorentz ${ }^{3}$ 2-manifold.

2. $S_{-b K I+b I I I}$ is the set of nonumbilic nonparabolic points of $S$ where $H \neq 0$. If $K$ is constant then $S_{-b I+b I I I}$ is flat.

Recall that minimal surface means an immersed surface with mean curvature $H \equiv 0$.

4.12. Corollary. If $(S, \nu)$ is an immersed minimal surface, then $S_{I I}$ is a flat Lorentz 2-manifold.

In the context of Corollaries 4.11 and 4.12 , we note that one combines (3.4) and (4.8) to see that $S_{I I}$ has connection form

$$
\beta_{12}=\frac{H}{|K|^{1 / 2}} \omega_{12} \text {. }
$$

${ }^{3}$ Here, Lorentz signature means that the pseudo-reimannian metric is neither positive definite nor negative definite.

\section{REFERENCES}

1. T. Klotz, Some uses of the second conformal structure on strictly convex surfaces, Proc. Amer. Math. Soc. 14 (1963), 793-799.

2. - Some geometric consequences of conformal structure, Trans. Amer. Math. Soc. 108 (1963), 38-53.

3. - - Another conformal structure on immersed surfaces of negative curvature, Pacific J. Math. 13 (1963), 1281-1288.

4. - Further geometric consequences of conformal structure, Trans. Amer. Math. Soc. 112 (1964), 67-78.

5. J. A. Wolf, Spaces of constant curvature, McGraw-Hill, New York (to appear).

The Institute for Advanced Study and

University of California, Berkeley 